\title{
Using neural networks for high-speed blood cell classification in a holographic-microscopy flow-cytometry system
}

\author{
B. Schneider* ${ }^{\mathrm{ab}}$, G. Vanmeerbeeck ${ }^{\mathrm{c}}$, R. Stahl ${ }^{\mathrm{c}}$, L. Lagae $^{\mathrm{cd}}$, P. Bienstman ${ }^{\mathrm{ab}}$
}

${ }^{a}$ Photonics Research Group (INTEC), Ghent University - imec, Sint-Pietersnieustraat 41, B-9000

Ghent, Belgium; ${ }^{b}$ Center for Nano- and Biophotonics (NB-Photonics), Ghent University, Sint-

Pietersnieuwstraat 41, B-9000 Ghent, Belgium; ${ }^{~}$ IMEC, Kapeldreef 75, B-3001 Leuven, Belgium;

${ }^{\mathrm{d}}$ INPAC and Dept. of Physics, KU Leuven, Celestijnenlaan 200 D, B-3001 Leuven, Belgium

\begin{abstract}
High-throughput cell sorting with flow cytometers is an important tool in modern clinical cell studies. Most cytometers use biomarkers that selectively bind to the cell, but induce significant changes in morphology and inner cell processes leading sometimes to its death. This makes label-based cell sorting schemes unsuitable for further investigation. We propose a label-free technique that uses a digital inline holographic microscopy for cell imaging and an integrated, optical neural network for high-speed classification. The perspective of dense integration makes it attractive to ultrafast, large-scale cell sorting. Network simulations for a ternary classification task (monocytes/granulocytes/lymphocytes) resulted in $89 \%$ accuracy.
\end{abstract}

Keywords: Digital inline holography, neural network, flow cytometry, cell sorting, integrated optics

\section{INTRODUCTION}

Modern clinical diagnostics heavily rely on human blood tests. Infectious diseases often find their counterpart in a changed composition of human whole blood, raising for example the presence of white blood cells (WBCs). These are of course not the only indicators but very significant ones. On the same line medical treatments and drug studies are commonly evaluated with respect to blood diagnostics. Therefore a reliable, precise, and fast characterisation of blood cells remains an ambitious goal challenging biomedical research.

Flow cytometers prevail as laboratory technique. Small-volume blood samples are prepared and loaded into the cytometer and the flow created therein ingeniously aligns the blood particles. This allows for accurate particle counting and characterisation at appreciable throughput rates. A short-coming or inconvenience might be that sometimes the sample preparation is rather involved invoking lysis and the attachment of biomarkers, fluorescent dies, or even magnetic beads to blood cells or cell components where they act as labels. As a consequence, most labelling techniques either induce a significant change in cell morphology or damage cell integrity causing its death. These side-effects often prohibit subsequent investigations of the functional cell and its environment.

It is thus desirable to develop label-free techniques that integrate easily with flow cytometry. A promising one, which is also used in fast particle volume tracking, is digital inline holography microscopy (DIHM) [1]. In this article, we propose the use of an optical neural network that classifies cells at a much shorter process delay. This is possible because the neural network takes the immediate holographic interference pattern as input. Neural networks have already been used in conjunction with cell type studies [2], [3], but to our knowledge its use for WBCs in combination with flow cytometry is reported for the first time. It promises an attractive approach to ultrafast, large-scale cell sorting ( $2 \mathrm{Mio}$. cells/sec) when densely integrated in a lab-on-chip configuration.

In the following section we discuss the working principle of the on-chip flow cytometer as it is conceived at imec [4]. Section III details the numerical implementation of the optical neural network and simulation results are presented and discussed in Section IV. 


\section{ON-CHIP FLOW CYTOMETER}

The current prototype of an on-chip flow cytometer cleverly combines three crucial functions: asserting a microfluidic flow in a narrow channel, microscopic imaging, and sorting (see Fig. 1).

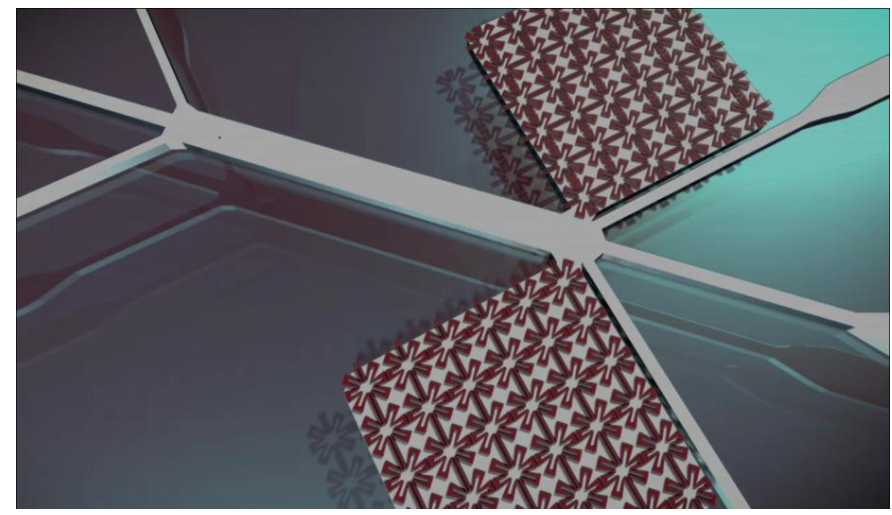

Figure 1. Typical design of a flow cytometer as proposed by imec [5]. Artistic view of the microfluidic channel (gray) and the steam bubble generating structure (red) next to the 3 outlets.

The microfluidic channel has an inlet where the loaded blood sample is dispensed. It aligns the cells so as to pass one by one the imaging area at a flow speed of $>1 \mathrm{~m} / \mathrm{sec}$. At the other end the channel displays multiple outlets which ought to collect the different cell types.

The imaging is based on a holographic method named DIHM. DIHM is a lens-free imaging technique and as such does not suffer from aberrations and other phase-perturbing imperfections. It relies on the known fact that a hologram contains all the necessary information of the wave-front emitted by the object under study which is encoded in its fringe pattern. An easy way to create these interference patterns is to coherently superpose a reference beam and the diffracted object beam. As the name of DIHM suggests this is done in line, meaning that the reference beam is equal to the object beam. This technique has already been proposed by Gabor [6], the inventor of holography, but encountered difficulties in its practical realization due to the twin image problem. For the flow cytometer at hand, the reference beam is simply the spherical wave emanating from a small pinhole on top of the microfluidic channel which is illuminated by a green laser. When the reference beam impinges on the transparent cell it is scattered into different directions. The scattered light and the reference beam overlap coherently at the bottom of the microfluidic channel where an image sensor is positioned. Since the sensor consists of a CCD-array the image is subsequently processed in digital form.

Once the decision was taken, the appropriate signal is sent to the switching unit prior to the outlets. The underlying switching mechanism is achieved by a jet of steam bubbles that deflect the bypassing cell into the desired outlet.

\section{NEURAL NETWORK IMPLEMENTATION AND SIMULATION}

Since the flow cytometer is devised on a silicon-on-insulator CMOS platform, we target the implementation of a neural network using CMOS compatible silicon photonics. The optical network is designed to work in the feed-forward configuration, thus avoiding any back-action of the output on its input. This simplifies the training of the neural network because techniques such as error back-propagation exist. One hidden layer of optical neurons is fit in between an input layer and an output layer. Fig. 2 shows a possible optical implementation. 


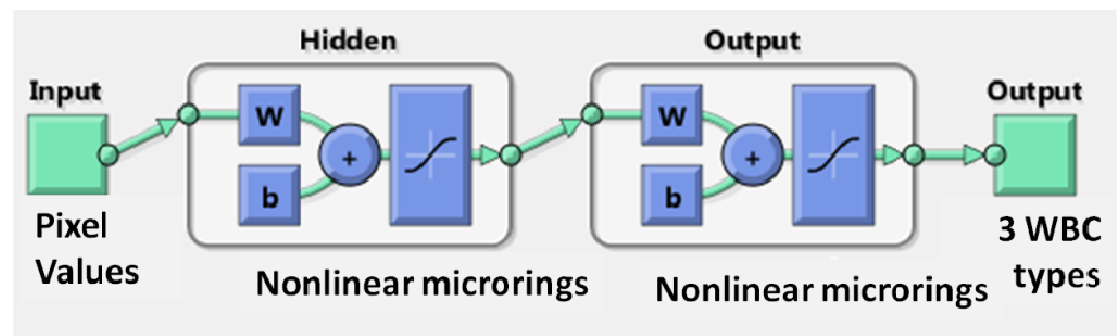

Figure 2. Schematic of the optical neural network [7] upon which the pattern detection depends. The pixel values from the input and can be realized by grating arrays. The nonlinear transmission function of each neuron in the hidden and output layer is realized by thermal microrings. Three different WBCs are distinguished at the output side.

The CCD image sensor is captures the diffracted as well as the reference field at the bottom of the microfluidic channel as a single cell is passing by. The weighting matrix combines the different input channels applying well designed weights to each of them. Each optical neuron receives input from a particular set of input nodes (gratings) and applies a nonlinear function to their sum. In our implementation we assume nonlinear thermal microrings as optical neuron units. Microrings form a resonator cavity with high quality factor at optical frequencies. A high quality factor allows for a high build-up of the optical power density which enables occurrence of multiple nonlinear effects. Among all the nonlinearities we are most interested in the self-heating of the cavity due to two-photon absorption and subsequent free carrier absorption. Eventually all the responses of the microring resonators are again linearly combined and nonlinearly transformed before being read out by an integrated photodetector array.

We simulated this neural network on real data which was collected with the flow cytometer, as described in section II. The dataset ( $\sim 6000$ cells) consists of three types of WBCs: granulocytes, lymphocytes, and monocytes. Their fractional occurrences in normal human blood are $65 \%, 30 \%$, and $5 \%$, respectively. We used 10 data folds in order to average over variations in the cell type distributions in training, validation, and test sets. A gradient-descent technique was implemented within the MATLAB ${ }^{\mathrm{TM}}$ Neural Network Toolbox [7] to update the network weights.

\section{SIMULATION RESULTS}

For the best neural net instantiation out of a random set of initial weights, we obtained the following classification result for the test set, shown below as a confusion matrix. The data is averaged over the 10 data folds. We notice that accuracies better than $90 \%$ are achieved which is in agreement with typical errors of about $10 \%$ (except for basophils) as is obtained by standard cytometers in clinical laboratories [8]. An increased error weight was introduced to compensate the skew in the dataset; the monocytes population is relatively scarce when compared to the two dominant WBC populations, granulocytes and lymphocytes. 


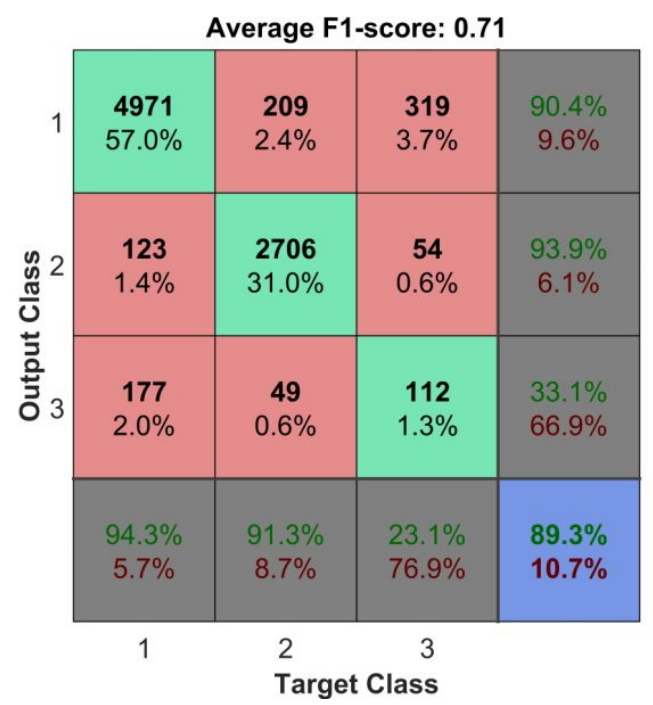

Figure 3. Confusion matrix for the three-part WBC classification. Target classes 1 to 3 represent granulocytes, lymphocytes, and monocytes. Total accuracy of nearly $90 \%$ is achieved.

\section{CONCLUSION}

Using numerical simulations, we confirmed successfully thatit is possible to use neural networks in flow cytometers when DIHM as imaging technique is used.

\section{REFERENCES}

[1] J. Garcia-Sucerquia, W. Xu, S. K. Jericho, P. Klages, M. H. Jericho, and H. J. Kreuzer, "Digital in-line holographic microscopy.," Appl. Opt., vol. 45, no. 5, pp. 836-50, 2006.

[2] J. Chen, Y. Zheng, Q. Tan, E. Shojaei-Baghini, Y. L. Zhang, J. Li, P. Prasad, L. You, X. Y. Wu, and Y. Sun, "Classification of cell types using a microfluidic device for mechanical and electrical measurement on single cells.," Lab Chip, vol. 11, no. 18, pp. 3174-3181, 2011.

[3] B. Swolin, P. Simonsson, S. Backman, I. Löfqvist, I. Bredin, and M. Johnsson, "Differential counting of blood leukocytes using automated microscopy and a decision support system based on artificial neural networks-evaluation of DiffMaster Octavia.," Clin. Lab. Haematol., vol. 25, no. 3, pp. 139-147, 2003.

[4] D. Vercruysse, A. Dusa, R. Stahl, G. Vanmeerbeeck, K. J. H. G. de Wijs, C. Liu, D. Prodanov, P. Peumans, and L. Lagae, "Three-part differential of unlabeled leukocytes with a compact lens-free imaging flow cytometer," Lab Chip, Dec. 2014.

[5] "IMEC On-Chip Single Cell Sorter | CytoFluidix.” [Online]. Available: http://www.cytofluidix.com/?p=162.

[6] D. GABOR, “A new microscopic principle.," Nature, vol. 161, no. 4098, p. 777, 1948.

[7] MATLAB and Neural Network Toolbox Release 2011b, The MathWorks Inc., Natick, Massachusetts, United States.

[8] J. L. Faucher, C. Lacronique-Gazaille, E. Frébet, F. Trimoreau, M. Donnard, D. Bordessoule, F. Lacombe, and J. Feuillard, “'6 Markers/5 colors' extended white blood cell differential by flow cytometry,” Cytom. Part A, vol. 71, no. 11, pp. 934-944, 2007. 